\title{
Quantitative Study on the Extinction Efficiency Error Caused by the Refractive Index During the Particulate Measurement
}

\author{
Houxin Cui
}

\author{
State Key Laboratory of Precision measuring technology and Instruments, Tianjin University, Tianjin 300072, P.R. \\ China
}

\begin{abstract}
The quantitative errors of extinction efficiency during the particulate measurement caused by the refractive index are studied. And the quantitative results are acquired when the real part of the refractive index and the imaginary part change respectively. The change ranges of the real part are $1+0.5 i$ to $2+0.5 i$ at a 0.1 interval and $1.5+0.5 i$ to $1.59+0.5 i$ at a 0.01 interval. Another the change ranges of the imaginary part are $1.57+0.1 i$ to $1.57+1 i$ at a $0.1 i$ interval and $1.57+1 i$ to $1.57+10 i$ at a $1 i$ interval. Firstly, the result shows the extinction efficiency change is linear with the real part change of the refractive index, but not linear with the imaginary part change. Secondly, when both the real part and the imaginary part change, the extinction efficiency will go to a constant value about 2.24 with the size parameter $x$ infinitely increasing. Thirdly, the errors are different with the change of $x$ when the real part changes. The maximal error value is $1.168 \%$ at $x=2$ and the end constant value is about $0.05 \%$. Also the extinction efficiency errors are $0.67 \%$ and $0.036 \%$ when the size of $x$ are 1 and 20 respectively when the imaginary part changes.
\end{abstract}

\section{INTRODUCTION}

During detecting the amount of the particulate in air, there are many factors that will put impact on the result, such as particle refractive index, particle size, temperature, pressure. Among these factors, the particle refractive index will contribute much to the measurement. In this paper, the quantitative errors of extinction efficiency during the particulate measurement caused by the refractive index are studied. And the quantitative results are acquired when the real part of the refractive index changes and the imaginary part changes.

Particulate material suspended in the atmosphere, commonly referred to as atmospheric aerosol, is produced by both nature and man. Naturally occurring materials include volcanic dust, meteoric dust, spores and seeds, particles of soil, sea salt etc. particle in the size range from 0.1 um to 10um produce significant optical effects in the atmosphere [1], also can cause many disease of body for example aspiratory illness, pulmonary disease, lung cancer, asthma [2] and so on. Research on the effects of particulate matter on human health was initiated in the 1970s $[3,4]$. So the determination of the amount of aerosols in the atmosphere has become a critical problem.

Many practical problems associated with the scattering of light radiation in the atmosphere require knowledge of the energy losses due to various scattering phenomena as well as that of the angular distribution and scattering radiation. The angular variation of the intensity of light scattered from atmospheric aerosols has been studied by a few earlier investigators and the capabilities of laser aerosol monitoring methods have been enhanced with the advent of the bistatic mode of operation of the lidar $[5,6]$.

*Address correspondence to this author at the State Key Laboratory of Precision measuring technology and Instruments, Tianjin University, Tianjin 300072, P.R. China; E-mail: tdchx@ hotmail.com
Generally, Beer-Lambert Law and Mie scattering theory are often used in the determination of the amount of aerosols. As Beer-Lambert Law:

$$
I=I_{0} e^{-\sigma_{e} L}
$$

where, I means the detected light energy with unit Watt, $I_{0}$ is the incident light energy with unit Watt, $\sigma_{e}$ is the extinction coefficient and $L$ is light path length with unit meter.

$$
\sigma_{e}=N A_{p} Q_{e}=\frac{\pi N d_{p}^{2} Q_{e}}{4}
$$

$N$ is the amount of aerosols, that is the concentration and $Q_{e}$ is the extinction efficiency.

$Q_{e}=I_{e} / I_{0}$

$I_{e}$ means the radiant power scattered and absorbed by a particle.

While, during detecting the amount of the particulate in air, there are many factors that will put impact on the result, such as particle refractive index, particle size, temperature, pressure. Among these factors, the particle refractive index will contribute much to the measurement. If the imaginary part of the refractive index increase, the contribution of light scattered twice predominates [7]. Maria et al. also found that by grain the larger the imaginary part of the refractive index, the smaller the energy density trapped within the grain [8]. However, it is not feasible to determine the refractive index of each individual particle within an aerosol population, an "average" or effective refractive index is normally used to represent the whole size distribution [9]. And according to $\mathrm{Li}$ [10], the large absolute value of imaginary part of refractive index in Minsk implies that the biomass burning aerosol from vegetation fires has stronger absorption than others. An increase in the imaginary part of the refractive index maintaining the real part constant produces and increase in the 
maximum of the linear polarization for incident unpolarized light [11].

In addition, Perrone shows that the backscatter coefficient of the particles are reduced as the imaginary part of the refractive index increases, where as extinction coefficients are weakly dependent on the refractive index [12]. And during the measurement of particles, for transparent particles, an increase in the particle refractive causes a decrease in the lower detection limit [13].

As we can see that the refractive index will take great impact on the measurement of particle concentration by using spectroscopy, so, in this paper, quantitative analysis will be studied about the effect caused by particle refractive index to the extinctive efficiency of the aerosols.

\section{METHOD}

Recently, Mie scattering theory has been widely used in the measurement of tiny particles, such as environment protection, energy, chronometer, weather and aerosol particulate. Note that:

$I=I_{0} e^{-\left(\alpha_{a}+\alpha_{S}\right) L}=I_{0} e^{-\alpha L}$

where $\alpha_{a}$ is absorption coefficient, $\alpha_{s}$ is the scattering coefficient, $\alpha$ is the total attenuate coefficient and $\alpha=\alpha_{a}+\alpha_{s}$, $L$ is the path length of the light source passing through the gas with particles.

While the absorption coefficient of suspended particulate matter can be relatively measured with the filter and pad technique [14,15], and inverse anomalous diffraction [16] models can be used to derive mean refractive index data for particle $[17,18]$. And the refractive index is the particle optical property relative to the atmosphere and can be used in the Mie scattering calculation to provide optical properties, typically denoted as:

$m(\lambda)=n(\lambda)+i k(\lambda)$

where $n$ is the real part of the index, $k$ is the imaginary part of the index or the absorption index [19], $\lambda$ is the incident light wavelength. According to Mie theory [16]:

$Q_{\text {ext }}=\frac{2}{x^{2}} \sum_{n=1}^{\infty}(2 n+1) \operatorname{Re}\left(a_{n}+b_{n}\right)$

All infinite series can be truncated after $n_{\max }$ terms. For this number Bohren and Huffman proposed the value

$n_{\max }=x+4 x^{1 / 3}+2$

And this value is used here as well. In equation, $x=k a$ is the size parameter, $a$ the radius of the spherical particle and $k=2 \pi / \lambda$ is the wave number and $\lambda$ the incident light wavelength.

\section{RESULT}

Two ranges of the real part of the particle refractive index will be selected in this paper. One varies from 1 to 2 at a 0.1 interval, and another ranges from 1.5 to 1.59 at a 0.01 interval. Fig. (1) shows the extinction efficiency change with the real part of refractive index in the range from $1+0.5 \mathrm{i}$ to $2+0.5 \mathrm{i}$ at a 0.1 interval with different $x$.

It can be seen from Fig. (1) that the extinction efficiency increases with the real part of the particle refractive index at a certain $x$, however the increased scope becomes smaller and smaller with the $x$ adding. Moreover, when the real part of refractive index is over than 1.3 , the extinction efficiency will go up first to the climax at about $x=3$ and then down. Finally, it will end at a constant value with the $x$ infinitely increasing, which is about 2.24.

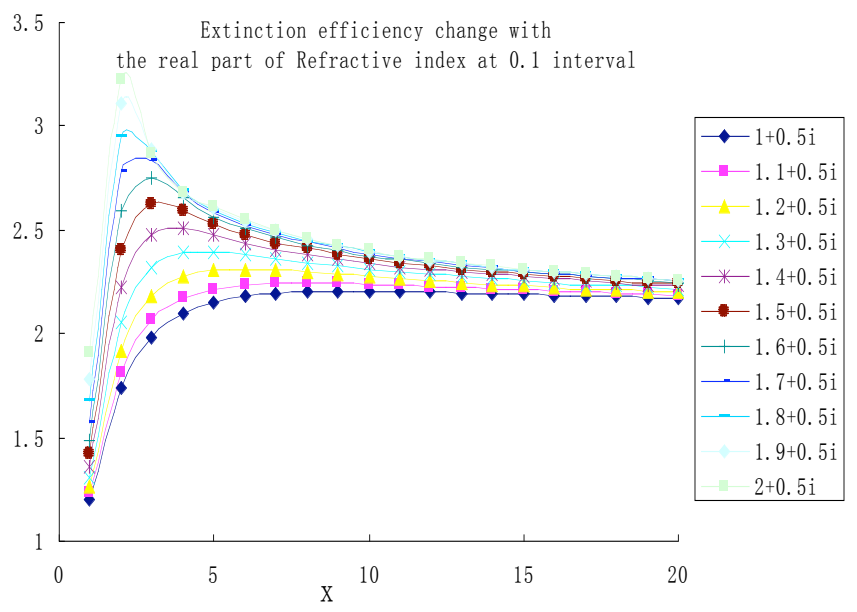

Fig. (1). Extinction efficiency change with the real part of the refractive index at 0.1 interval.

In another range of the real part of refractive index, from $1.5+0.5 i$ to $1.59+0.5 i$, the extinction efficiency changes with the real part are shown as Fig. (2). And the right picture denotes the amplificatory part in the local box of the left figure, which is labeled in Fig. (2). From Fig. (2), the extinction efficiency also becomes increasing with the real part of the refractive index. And just as same as in the range from $1+0.5 i$ to $2+0.5 i$ at a 0.1 interval, the extinction efficiency will go up first to the climax at about $x=3$ and then down till reach a constant value.

Then, the change tendency of the extinction efficiency at $x=3$ can be drawn as Fig. (3), from which it can be seen that the extinction efficiency change is linear with the real part, and so do at other $x$.

Therefore, the quantitative error caused by the bias of the real part of the refractive index can be calculated and shown as in Fig. (4). In this figure, all error data are computed when the real part of the refractive index varies $1 \%$. And according to Fig. (4), the errors are different with the change of $x$. they ascend first and then descend, and will go to a constant value with the infinite $x$. the maximal error value is $1.168 \%$ at $x=2$ and the end constant value is about $0.05 \%$.

Just like in Section 3.1, two ranges of the imaginary part of the particle refractive index will be selected. One varies from $0.1 i$ to $1 \mathrm{i}$ at a $0.1 i$ interval, and another ranges from $1 i$ to $10 i$ at a $1 i$ interval. Fig. (5) shows the extinction efficiency change with the imaginary part of refractive index in the range from $1.57+0.1 i$ to $1.57+i$ at a $0.1 i$ interval 

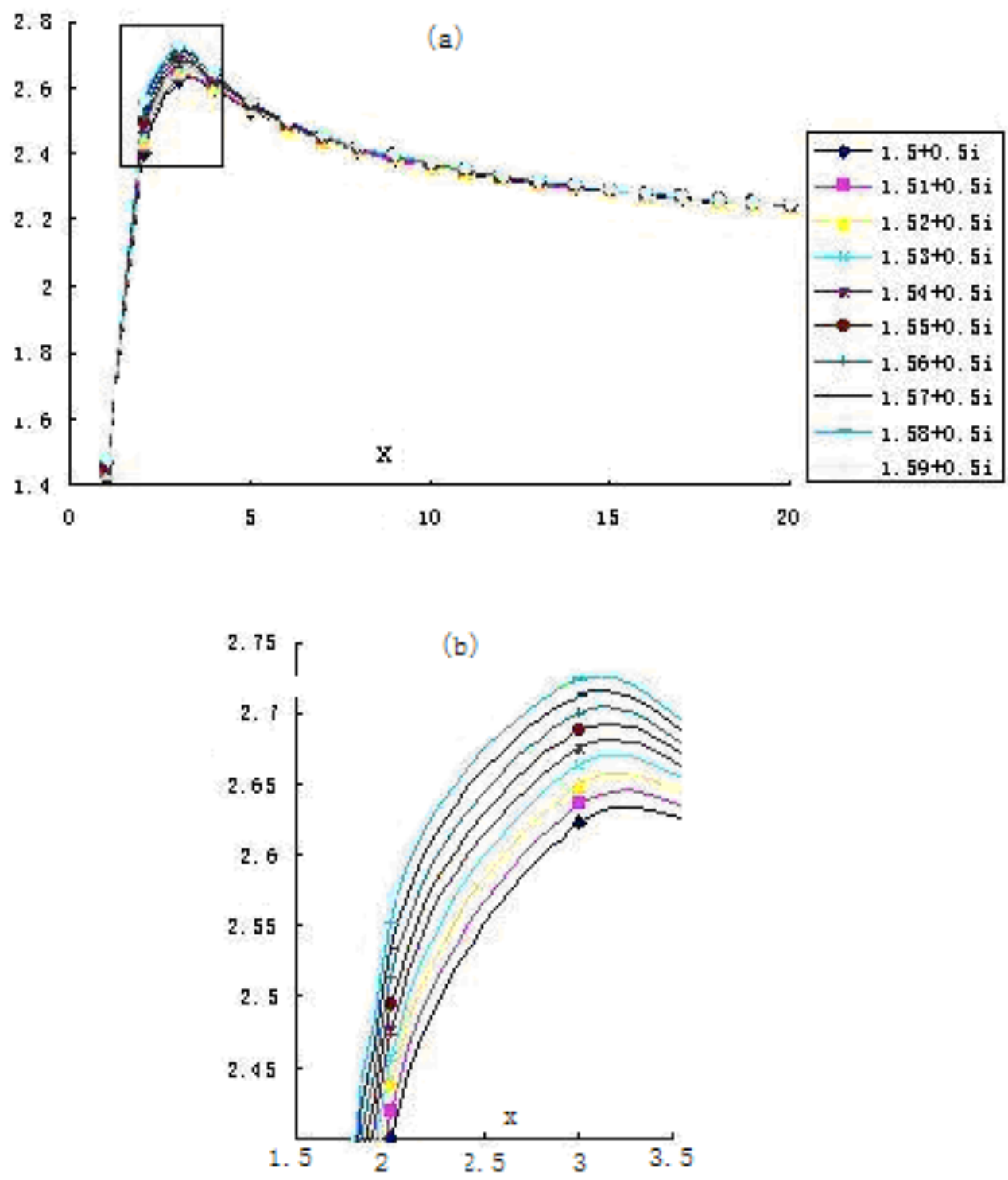

Fig. (2). Extinction efficiency change with the real part of the refractive index at 0.01 interval (the right picture denotes the amplificatory part in the local box of the left figure).

with different $x$. In addition, four different particle sizes, for example $x=5,10,15,20$, are selected to show the extinction efficiency change with the imaginary part of the refractive index, as shown in Fig. (6).

It can be seen from Figs. $(\mathbf{5 , 6})$ that the extinction efficiency does not change linearly with the imaginary part of the particle refractive index at certain $x$. With the imaginary part of the refractive index increasing, the extinction efficiency will fall down first and then rise up at about $\mathrm{m}=1.57+0.5 i$.

Moreover, to all the imaginary parts, the extinction efficiency will go up first to the climax at about $x=3$ and then down. Finally, it will end at a constant value with the $x$ infinitely increasing.

In another range of the real part of refractive index, from $1.57+1 i$ to $1.57+10 i$, the extinction efficiency changes with the imaginary part are shown as Fig. (7).

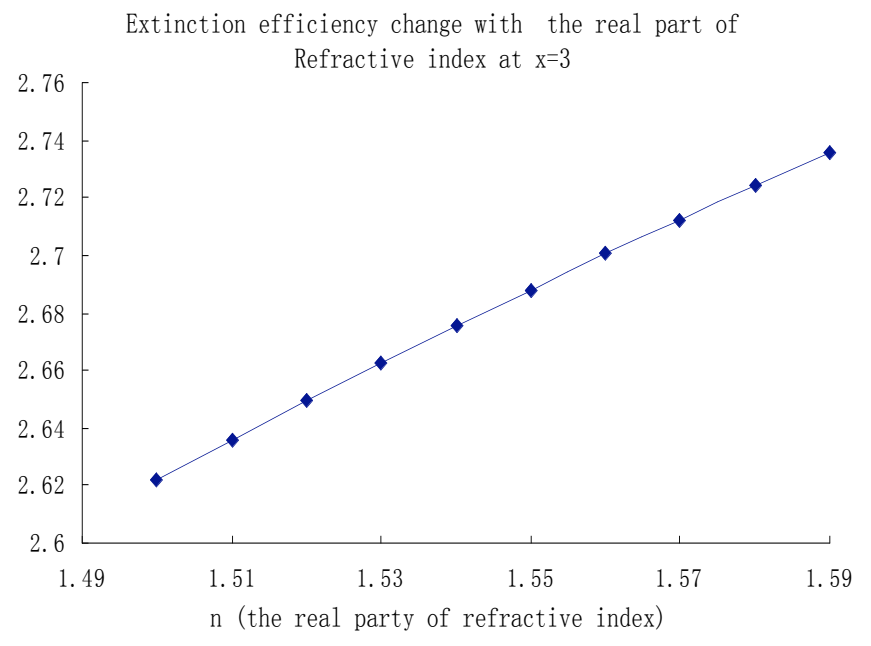

Fig. (3). Extinction efficiency change with $x=3$. 


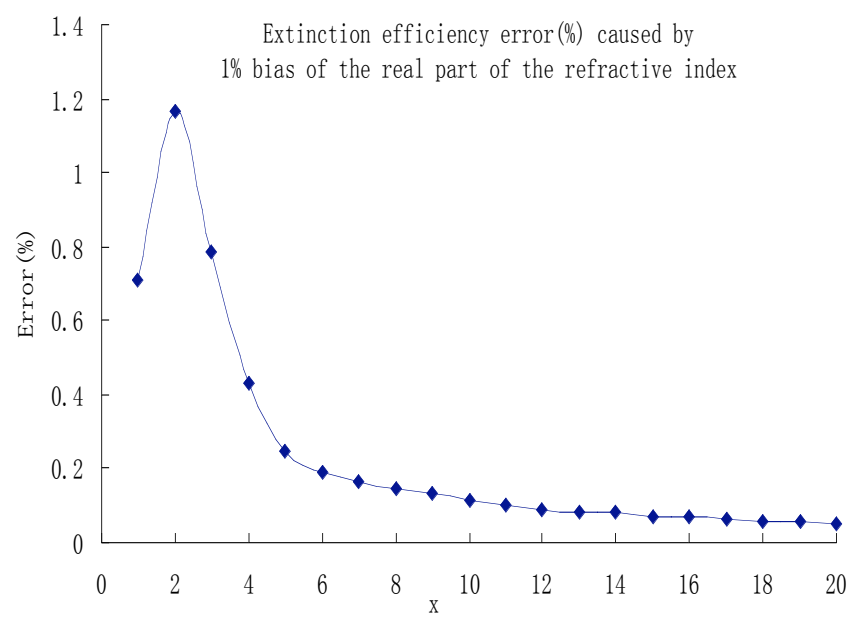

Fig. (4). Quantitative error(\%) at different $x$.

According to this figure, the extinction efficiency also varies nonlinearly with the imaginary part of the refractive index, just like in the range from $0.1 i$ to $1 i$. But the difference is that the extinction efficiency will decrease all long with the $x$ infinitely increasing, till to a constant value about 2.2 .

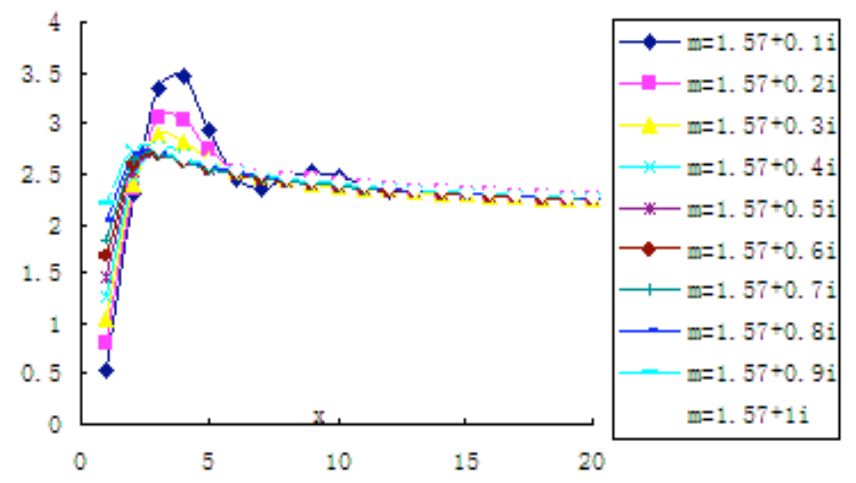

Fig. (5). Extinction efficiency change with the imaginary part of the refractive index at $0.1 i$ interval.

Therefore, the quantitative error caused by the bias of the imaginary part of the refractive index can be calculated and shown as in Fig. (8). In this figure, all error data at different $x$ are computed when the real part of the refractive index varies $1 \%$. And according to Fig. (8), the errors are different with the change of $x$ and the bigger the size $x$, the smaller the error. The extinction efficiency errors are $0.67 \%$ and $0.036 \%$ when the size $x$ are 1 and 20 respectively.

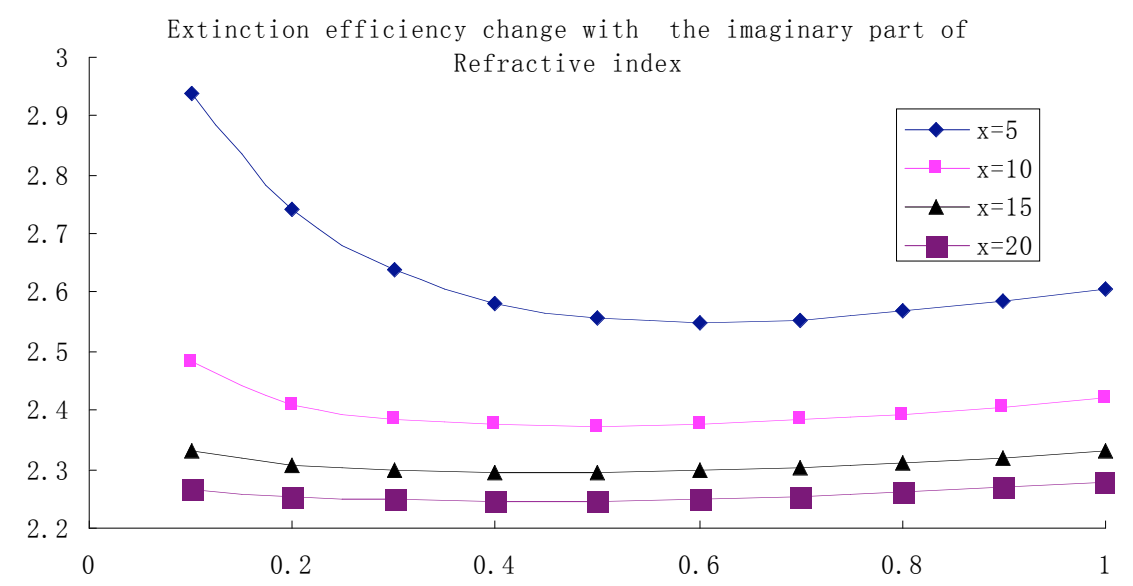

Fig. (6). Extinction efficiency change with the imaginary part of the refractive index at $0.1 i$ interval with $\mathrm{x}=5,10,15,20$.

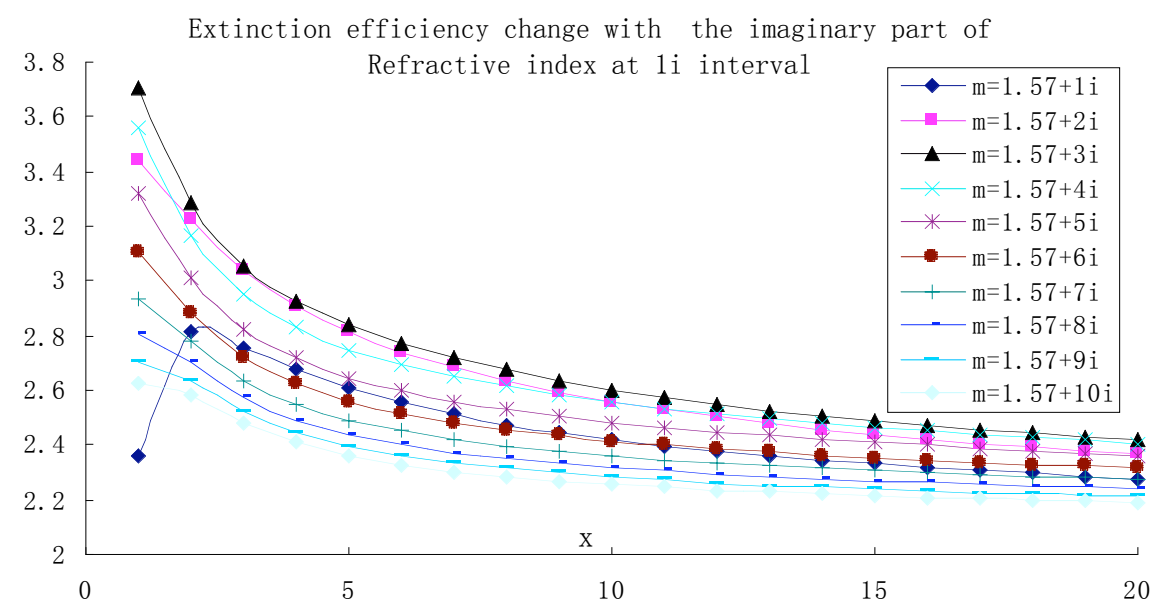

Fig. (7). Extinction efficiency change with the imaginary part of the refractive index at $1 i$ interval. 


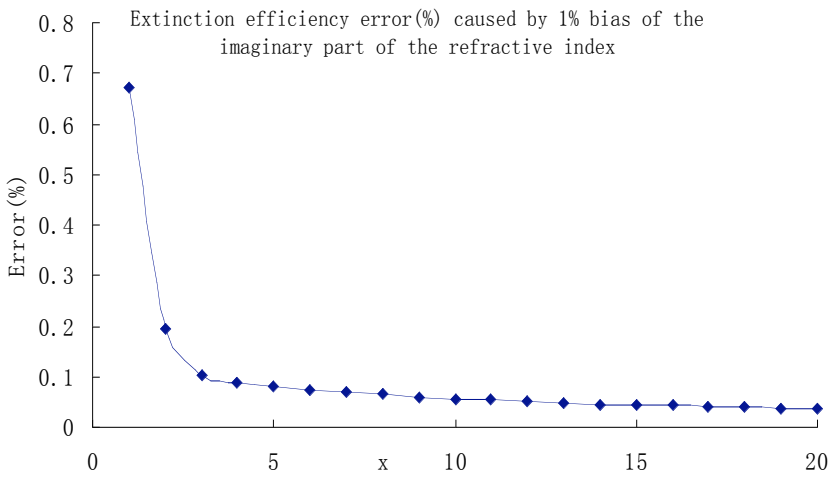

Fig. (8). Quantitative error(\%) of extinction efficiency caused by $1 \%$ bias of the imaginary part of the refractive index.

\section{CONCLUSION}

Firstly, it can be seen from the result data that the extinction efficiency change is linear with the real part change of the refractive index, but not linear with the imaginary part change. Secondly, to the changes of both the real part and the imaginary part, the extinction efficiency will go to a constant value about 2.24 with the $x$ infinitely increasing. Thirdly, to the change of the real part, the errors are different with the change of $x$. They ascend first and then descend till to a constant with the infinite $x$. The maximal error value is $1.168 \%$ at $x=2$ and the end constant value is about $0.05 \%$. However to the change of the imaginary part, the result is the bigger the size of $x$, the smaller the error. The extinction efficiency errors are $0.67 \%$ and $0.036 \%$ when the size of $x$ are 1 and 20 respectively.

\section{ACKNOWLEDGEMENT}

This work was supported by the Natural Science Foundation of Tianjin (No. 06YFJMJC06700).

\section{REFERENCES}

Raj PE, Devara PC, Pandithurai G, Maheskumar RS, Dani KK. "Some atmospheric aerosol characteristics as determined from laser angular scattering measurements at a continental urban station", atmósfera, vol.17, P.39-52, Jan 2004.

[2] Chambellan A, Crestani B, Soler P. "Diesel Particles and Allergy: Cellular Mechanisms", Allergy Immunol (Paris), vol.32, P. 43-48, Feb 2000.

[3] Larsen RI. "Relating air pollutant effects to concentration and control”, Air Pollution Control Assoc, vol.20, P.214, May 1970.
[4] Holland WW, Bennett AE, Cameron IR. "Health effects of particulate pollution: reappraising the evidence", J. Epidemiol, vol.110, P. 527-558, Nov 1979.

[5] Pandithurai G, Devara PCS, Raj PE. "Aerosol size distribution and refractive index from bistatic lidar angular scattering measurements in the surface layer", Romote sensing Environ., vol.56, P. 87-96, May 1996.

[6] Ward G, Cushing KM, Mcpeters RD, Green AES. "Atmospheric aerosol index of refraction and size-altitude distribution from bistatic laser scattering and solar aureole measurements", Appl. Opt., vol.12, P. 2585-2592, Nov 1973.

[7] Tishkovets VP, Petrova EV, Jockers K. "Optical properties of aggregate particles comparable in size to the wavelength", Journal of Quantitative Spectroscopy \& Radiative Transfer, vol.86, P. 241265, Jul 2004.

[8] Iati MA, Saija R, Giusto A, Denti P, Borghese F. "Light within small particles", Journal of Quantitative Spectroscopy \& Radiative Transfer, vol.100, P. 157-164, Aug 2006.

[9] Guyon P, Boucher O, Graham B, Beck J, Mayol-Bracero OL. "Refractive index of aerosol particles over the Amazon tropical forest during LBA-EUSTACH 1999", Aerosol Science, vol.34, P. 883-907, Jul 2003

[10] Li ZQ, Goloub P, Devaux C, Gu XF, Deuzé JL, Qiao YL, Zhao FS. "Pretrieval of aerosol optical and physical properties from groundbased spectral multi-angular and polarized sun-photometer measurements", Remote Sensing of Environment, vol.101, P. 519-533, Apr 2006.

[11] Vilaplana R, Moreno F, Molina A. "Study of the sensitivity of sizeaveraged scattering matrix elements of nonspherical particles to changes in shape porosity and refractive index", Journal of Quantitative Spectroscopy \& Radiative Transfer, vol.100, P.415-428, Jul 2006.

[12] Perrone MR, Barnaba F, De Tomasi F, Gobbi GP, Tafuro AM. "Imaginary refractive-index effects on desert-aerosol extinction versus backscatter relationships at $351 \mathrm{~nm}$ : numerical computations and comparison with Raman lidar measurements", Applied Optics, vol.43, P. 5531-5541, Oct 2004

[13] Yoo SH, Chae SK, Liu BYH. "Influence of particle refractive index on the lower detection limit of light scattering aerosol counters", Aerosol Science and Technology, vol.25, P. 1-10, Jan 1996.

[14] Yentsch CS. "Measurement of visible light absorption by particulate matter in the ocean", Limnol. Oceanogr., vol.7, P. 207-217, Mar 1962.

[15] Mitchell BG. "Algorithms for determining the absorption coefficient of aquatic particulates using the quantitative filter technique (QFT)", SPIE, vol.1302, P. 137-148, 1990.

[16] Van de Hulst HC. Light Scattering by Small Particles; Wiley: New York, USA1957.

[17] Bricaud A. "Morel A., Light attenuation and scattering by phytoplanktonic cells: a theoretical modeling", Appl. Opt. vol.25, P. 571-580, Feb 1986.

[18] Stramski D, Morel A, Bricaud A. "Modelling the light attenuation and scattering by spherical phytoplankton cells: a retrieval of the bulk refractive index", Appl. Opt., vol.27, P. 3954-3956, Oct 1988.

[19] Aas E. "Refractive index of phytoplankton derived from its metabolite composition", J. Plank. Res., vol.18, P. 2223-2249, Dec 1996. 